\title{
Consistency of Ocular Palpation Tonometry with Goldmann Applanation Tonometry and its Reproducibility by Separate Examiners
}

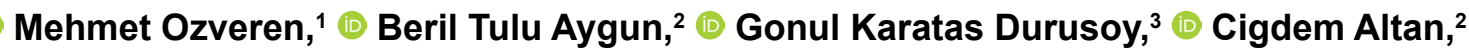 \\ Muhittin Taskapili² \\ ${ }^{1}$ Department of Ophthalmology, Edirne Sultan $1^{\text {st }}$ Murat State Hospital, Edirne, Turkey \\ ${ }^{2}$ Department of Ophthalmology, Beyoglu Eye Training and Research Hospital, Istanbul, Turkey \\ ${ }^{3}$ Department of Ophthalmology, Gaziantep Ersin Arslan Training and Research Hospital, Gaziantep, Turkey
}

\begin{abstract}
Objectives: Elevated intraocular pressure (IOP) remains to be the best known modifiable risk factor for glaucoma. Ocular palpation tonometry (OPT) is an old, fast, and simple method for IOP measurement. The aim is to investigate accuracy, reproducibility of OPT, and its suitability to be used for screening purposes.

Methods: Consecutive patients without corneal pathology and glaucoma diagnosis history were included in the study. After routine ophthalmologic examination, the IOP was measured by an ophthalmology specialist with Goldmann applanation tonometry (GAT). Later, a blinded other ophthalmology specialist and a resident evaluated the same patients' IOP with OPT. Agreement between GAT and OPT and reproducibility of OPT were evaluated using Cohen's kappa analysis.

Results: A total of 214 eyes of 107 patients were included in the study with a mean IOP of $16.4 \pm 3.6$ (min: 8 , max: 27) $\mathrm{mmHg}$ with GAT. OPT measurements by the resident ophthalmologist and the ophthalmology specialist were in concordance with GAT for $44.4 \%$ and $50.5 \%$ of the evaluations, respectively. We could not detect a significant kappa value for the agreement between GAT and OPT measurements by the resident ophthalmologist $(p=0.333)$, whereas a slight agreement was found between GAT and OPT measurements by the ophthalmology specialist $(k=0.176, p=0.001)$. OPT measurements showed slight reproducibility $(k=0.153, p=0.009)$.

Conclusion: OPT has low reliability and reproducibility and is not suitable to be used as a screening test. Except obligatory states, it should not be used as a primary and only test and must be verified by other methods.

Keywords: Glaucoma screening, intraocular pressure, ocular palpation tonometry, tactile tonometry.
\end{abstract}

\section{Introduction}

Glaucoma is one of the leading causes of blindness worldwide, and high intraocular pressure (IOP) is the most important risk factor known to cause glaucoma $(I, 2)$. Currently, Goldmann applanation tonometry (GAT) is the gold standard and reference method for IOP measurement (3).

Although GAT is commonly being used in developed and developing countries, some specific situations and diseases still entail using other methods including subjective ones. Furthermore, in underdeveloped countries and in some centers of developing countries, GAT equipment may not be readily available. Other options of tonometry are used for intraoperative IOP measurement, for uncooperative patients, kids, and patients who cannot sit for biomicroscopic evaluation because GAT is impossible or impracticable for these groups. Furthermore, GAT is known to give inaccurate

\section{Address for correspondence: Mehmet Ozveren, MD. Edirne Sultan 1. Murat Devlet Hastanesi, Oftalmoloji Anabilim Dalı, Edirne, Turkey \\ Phone: +90 5554172949 E-mail: ozveren.mehmet@gmail.com}

Submitted Date: April 28, 2018 Accepted Date: November 01, 2018 Available Online Date: December 24, 2018

${ }^{\circ}$ Copyright 2018 by Beyoglu Eye Training and Research Hospital - Available online at www.beyoglueye.com 
results for patients with corneal scar or edema and patients with corneal transplantation, so other tonometry options are also commonly used for these patient groups $(4,5)$. Ocular palpation tonometry (OPT) (digital tonometry, tactile tonometry, and IOP palpation) technique is a fast, cheap, and practical method which is commonly used for IOP measurement for the above-mentioned situations and for screening purposes (6).

Some studies analyzing the accuracy of OPT reported high accuracy rates when performed by experienced hands (7), whereas other studies reported poor accuracy except for very high IOP values (8).

Some investigators focused on the hypothesis that accuracy of OPT can be enhanced by practice and experience and claimed that OPT can be enhanced enough to be used with high accuracy $(4,7)$. In literature, some reports suggested that the blind people may be more successful to perform OPT due to their enhanced tactile perceptions and these people can be employed for IOP screening (9).

In this study, we aimed to evaluate the accuracy, reproducibility of OPT, and its feasibility as a screening method.

\section{Methods}

Consecutive patients who applied to our outpatient clinic with complaints such as refractive errors between August I, 2016, and September 30, 2016, were included in the study. Age under 40, any type of corneal pathology, and diagnosed or suspected glaucoma were chosen as exclusion criteria. This study was performed in accordance with the Helsinki Declaration, and patients' informed consents were obtained.

GAT measurements were performed and recorded by one ophthalmology specialist for all eyes. Later, a blinded other ophthalmology specialist and a blinded ophthalmology resident used OPT to define IOP in one of the following IOP ranges: " $\leq 10 \mathrm{mmHg}, 1 \mathrm{I}-15 \mathrm{mmHg}, 16-20 \mathrm{mmHg}, 2 \mathrm{I}-25$ $\mathrm{mmHg}, 26-30 \mathrm{mmHg}$, and $\geq 30 \mathrm{mmHg}$." OPT measurements were performed with index fingers over eyelids of patients who were instructed to look down during measurements. Furthermore, age, sex, central corneal thickness (CCT), and spherical equivalent values were recorded for all patients. CCT measurements were done with non-contact specular microscope (Nidek Co., Ltd., Gamagori, Japan, CEM-530).

Cohen Kappa test was used to assess OPT reproducibility by two separate examiners and, OPT and GAT concordance. Kappa values were interpreted according to guidelines defined by Landis and Koch (10). Spearman test and ordinal regression analyses were used to assess the effect of age, sex, CCT, and spherical equivalent OPT and GAT concordance. Statistical significance was accepted when $\mathrm{P}<0.05$.

\section{Results}

A total of 214 eyes of 107 patients were included in the study. There were 50 male $(46.7 \%)$ and 57 female $(53.3 \%)$ patients with a mean age of $55.2 \pm 10.5$ years.

Mean IOP detected by GAT was $16.4 \pm 3.6$ (min: 8 , max: 27) $\mathrm{mmHg}$, mean CCT was $556 \pm 4 \mathrm{I} \mathrm{Mm}$, and mean spherical equivalent was $-0.14 \pm 2.68 \mathrm{D}$. None of the eyes was classified as $>30 \mathrm{mmHg}$ with OPT method by either physician. Furthermore, there were no eyes with GAT measurement over $30 \mathrm{mmHg}$.

We detected that OPT performed by the resident ophthalmologist and the ophthalmology specialist was in concordance with GAT values for $44.4 \%$ and $50.5 \%$ of the eyes tested, respectively. The agreement and disagreement percentages of GAT with OPT performed by ophthalmology resident and specialist are presented in Table I which was classified according to GAT values.

We could not detect a significant kappa value for the agreement between GAT measurements and OPT measurements performed by the resident ophthalmologist $(p=0.333)$. We detected a slight agreement between GAT measurements and OPT measurements performed by the ophthalmology specialist $(k=0.176, p=0.001)$. OPA measurements performed by the ophthalmology specialist and resident also showed slight agreement $(k=0.153, p=0.009)$.

When the eyes were divided into two groups according to IOP detected by GAT, OPT performed by the resident and the specialist was in concordance with GAT values for $84.6 \%$ and $87.4 \%$ of the eyes tested, respectively (Table 2 ). We could not detect a significant kappa value for the agreement between GAT measurements and OPT measurements performed by the resident ophthalmologist $(p=0.992)$. We detected a fair agreement between GAT measurements and OPT measurements performed by the ophthalmology specialist $(k=0.217, p<0.00 \mathrm{I})$. OPA measurements performed by the ophthalmology specialist and resident also showed slight agreement $(k=0.134, p=0.005)$.

There was no significant correlation between OPT states according to GAT (overestimation, agreement, and underestimation) and age, spherical equivalent, and sex parameters for both resident and specialist measurements $(p>0.05)$. However, CCT (Spearman-resident ophthalmologist: -0.364 and specialist: -0.400 ) and GAT measurements showed significant negative correlation (Spearman-resident ophthalmologist: -0.644 and specialist: $-0.60 \mathrm{I}$ ) with OPT state according to GAT $(p<0.001)$, i.e., as CCT and GAT values increase, the probability of overestimation by OPT decreases, whereas underestimation probability increases. Following ordinal regression analysis provided that GAT measurement preserved significance $(p<0.00 \mathrm{I})$, whereas CCT lost significance. 


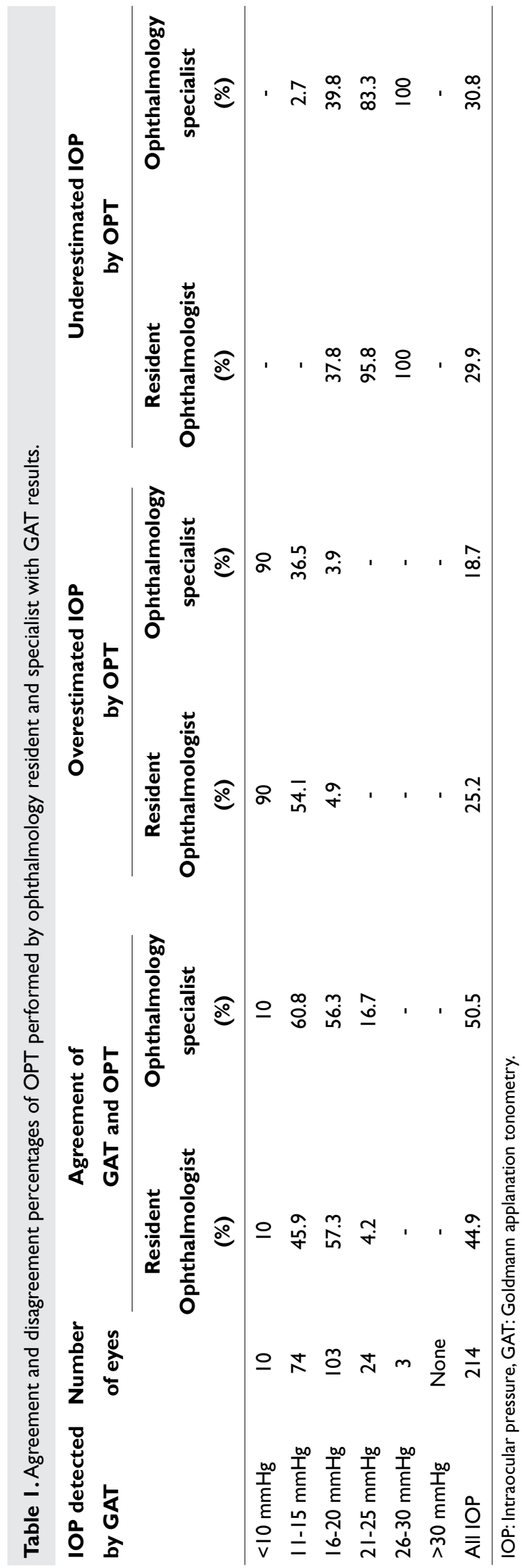

Table 2. Agreement of OPT and GAT when eyes were grouped according to GAT measurements above and below $21 \mathrm{~mm} \mathrm{Hg}$.

\begin{tabular}{|c|c|c|}
\hline \multirow{2}{*}{$\begin{array}{l}\text { IOP detected } \\
\text { by GAT }\end{array}$} & \multicolumn{2}{|c|}{ Agreement of GAT and OPT } \\
\hline & $\begin{array}{c}\text { Resident } \\
\text { Ophthalmologist (\%) }\end{array}$ & $\begin{array}{c}\text { Ophthalmology } \\
\text { specialist (\%) }\end{array}$ \\
\hline$<21 \mathrm{mmHg}(187)$ & 96.3 & 97.3 \\
\hline$\geq 21 \mathrm{mmHg}(27)$ & 3.7 & 18.5 \\
\hline All eyes $(2 \mid 4)$ & 84.6 & 87.4 \\
\hline
\end{tabular}

IOP: Intraocular pressure; GAT: Goldmann applanation tonometry.

\section{Discussion}

In this study, our purpose was to evaluate the accuracy of OPT in comparison to GAT, reproducibility of OPT by different examiners, and its feasibility to be used as a ocular hypertension screening method. Birnbach and Leen (7) conducted a study on cadaveric eyes; they yielded IOPs ranging between 5 and $40 \mathrm{mmHg}$ at intervals of $5 \mathrm{mmHg}$. Then, one experienced and one inexperienced investigator tried to estimate the IOP by palpating the cadaveric cornea. The experienced investigator was able to detect IOP accurately for $46 \%$ of cases and within a deviation of $5 \mathrm{mmHg}$ for $100 \%$ of cases. Inexperienced investigator was able to detect IOP accurately for $21 \%$ of cases and within a deviation of $5 \mathrm{mmHg}$ for $62 \%$ of cases. In our study, the ophthalmology specialist and resident were able to detect IOP accurately for $50.5 \%$ and $44.9 \%$ of cases, respectively. Our results are comparable to results of Birnbach's study, in which bare cornea was palpated for IOP estimation unlike our work.

Baum et al. (8) reported a low correlation between tactile IOP measurement and GAT but reported some success at IOPs over $30 \mathrm{mmHg}$. The reported low success of Baum's study was in agreement with our study. Unlike Baum's study, there were no patient in our study with IOP over $30 \mathrm{mmHg}$.

In another study, OPT and MacKay-Marg tonometer measurements were compared in eyes after keratoplasty. In that study, OPT measurements were found to be $5 \mathrm{mmHg}$ higher than MacKay tonometry measurements, and it was reported that, for some doctors and for some patients, OPT method may be of value to practice (II). High OPT measurements compared to the control tonometer method in that study were considered to be due to bias created by post-operative high IOP expectancy, post-operative eyelid edema, and eye tenderness. The use of different groups of patients in different studies directly affects the expected IOP value which in turn directly has an effect on the results obtained by this subjective method through bias.

When IOP was classified into five groups $(\leq 10 \mathrm{mmHg}$, II-15 mmHg, 16-20 mmHg, 2I-25 mmHg, $26-30 \mathrm{mmHg}$, and $\geq 30 \mathrm{mmHg}$ ), agreement of OPT measurements by oph- 
thalmology specialist and resident with GAT was $50.5 \%$ and $44.4 \%$, respectively. When IOP was classified into two groups $(\geq 21 \mathrm{mmHg}$ and $<2 \mathrm{I} \mathrm{mmHg}$ ), agreements were $87.4 \%$ and $84.6 \%$, respectively. Despite these relatively high agreement rates, Cohen's kappa test revealed slight-fair agreement between OPT and GAT and also between OPT measurements of different ophthalmologists. These results suggest that the tactile tonometry method has low accuracy and reproducibility.

Cohen's Kappa test is used to test agreement between two tests or two examiners for qualitative items (I2). It is generally thought to be a more robust measure than simple percent agreement calculation since $\mathrm{K}$ takes into account the possibility of the agreement occurring by chance (I2). Some IOP intervals in the normal population are seen more frequently, and the results reported by physicians who know that the normal population is being tested are also concentrated in the frequent IOP values so that expectation bias probably caused relatively high agreement percentage ratios in our study.

The mean IOP in the study population was $16.4 \pm 3.6$ $\mathrm{mmHg}$ and was found to be slightly higher than the mean IOP $(15.4 \mathrm{mmHg})$ determined in the study of the Beaver Dam with similar age range (I3).

The fact that most of the IOPs of the patients are anticipated to be close to the mean IOP of the population because the patients included in the study are patients without ocular hypertension history is a limitation of the study which can cause bias in OPT measurements. Significant negative correlations were found between GAT measurements and states of OPT measurements (low, high, or agreement) compared to GAT. That is, the probability of underestimation by OPT increases as the GAT measurement value increases. In the patient group with GAT detected IOP values $\geq 2 \mathrm{I} \mathrm{mmH}$, ophthalmology specialist and residents reported results below $21 \mathrm{mmHg}$ for $81 \%$ and $96.3 \%$ of the eyes, respectively. In this group, which is important for high IOP screening, lower values detected by OPT than the actual values indicate that the use of this tonometry method for screening is not appropriate.

In conclusion, OPT has low reliability and is not recommended as a screening test because it is inefficient in detecting slight moderate IOP elevations frequently seen in earlystage glaucoma disease. Unless imperative, its results should not be relied on and must be verified by other methods.

\section{Disclosures}

Peer-review: Externally peer-reviewed.

Conflict of Interest: None declared.

Authorship Contributions: Involved in design and conduct of the study (MO, BTA, GKD); preparation and review of the study (MO, BTA, GKD, CA, MT); data collection (MO, BTA); and statistical analysis (MO).

\section{References}

I. Bourne RR, Stevens GA, White RA, Smith JL, Flaxman SR, Price $\mathrm{H}$, et al.; Vision Loss Expert Group. Causes of vision loss worldwide, 1990-2010: a systematic analysis. Lancet Glob Health 20I3; I:e339-49.

2. Kanski JJ, Bowling B, Nischal KK, Pearson A. Clinical ophthalmology: a systematic approach. 7th ed. New York: Elsevier/ Saunders; 20II.

3. Nuyen B, Mansouri K. Fundamentals and Advances in Tonometry. Asia Pac J Ophthalmol (Phila) 2015;4:66-75.

4. Heidary R, Heidary F, Rahimi A, Gharebaghi R. An innovative educational model in intraocular pressure measurement. Med Hypothesis Discov Innov Ophthalmol 20I2; I:50-I.

5. Frenkel S, Katz S, Horani A, Kaiserman I, Asleh SA, Blumenthal EZ. Estimating intraocular pressure using a glass rod. Ann Ophthalmol (Skokie) 2006;38:195-9.

6. Polyvás PP, Peyman G, Enikov ET. Trans-scleral tactile tonometry: an instrumented approach. Med Eng Phys 2013;35:937-43.

7. Birnbach CD, Leen MM. Digital palpation of intraocular pressure. Ophthalmic Surg Lasers 1998;29:754-7.

8. Baum J, Chaturvedi N, Netland PA, Dreyer EB. Assessment of intraocular pressure by palpation. Am J Ophthalmol 1995; I 19:650-I.

9. Heidary F, Gharebaghi R, Heidary R. Palpation by blind examiners: A novel approach for glaucoma screening. Clin Ophthalmol 2010;4:67I-2.

10. Landis JR, Koch GG. The measurement of observer agreement for categorical data. Biometrics 1977;33:I59-74.

I I. Rubinfeld RS, Cohen EJ, Laibson PR, Arentsen JJ, Lugo M, Genvert Gl. The accuracy of finger tension for estimating intraocular pressure after penetrating keratoplasty. Ophthalmic Surg Lasers 1998;29:213-5.

12. McHugh ML. Interrater reliability: the kappa statistic. Biochem Med (Zagreb) 2012;22:276-82.

13. Klein BE, Klein R, Linton KL. Intraocular pressure in an American community. The Beaver Dam Eye Study. Invest Ophthalmol Vis Sci 1992;33:2224-8. 